Research Article

\title{
Electrochemical Characteristics Based on Skin-Electrode Contact Pressure for Dry Biomedical Electrodes and the Application to Wearable ECG Signal Acquisition
}

\author{
Jinzhong Song $\mathbb{D},{ }^{1,2}$ Tianshu Zhou $\mathbb{D}^{3},{ }^{3}$ Zhonggang Liang, ${ }^{2}$ Ruoxi Liu, ${ }^{4}$ Jianping Guo, ${ }^{2}$ \\ Xinming Yu, ${ }^{2}$ Zhongping Cao, ${ }^{2}$ Chuang Yu, ${ }^{2}$ Qingjun Liu, ${ }^{1}$ and Jingsong $\mathrm{Li}\left(\mathbb{C}^{1,3}\right.$ \\ ${ }^{1}$ Key Laboratory for Biomedical Engineering of Ministry of Education, Engineering Research Center of EMR and Intelligent \\ Expert System, Ministry of Education, College of Biomedical Engineering and Instrument Science, Zhejiang University, \\ Hangzhou 310027, China \\ ${ }^{2}$ State Key Laboratory of Space Medicine Fundamentals and Application, China Astronaut Research and Training Center, \\ Beijing 100094, China \\ ${ }^{3}$ Research Center for Healthcare Data Science, Zhejiang Lab, Hangzhou 311100, China \\ ${ }^{4}$ Pony Testing International Group Co. Ltd, Zhejiang Lab, Beijing 100095, China \\ Correspondence should be addressed to Jingsong Li; ljs@zju.edu.cn
}

Received 25 June 2021; Revised 15 August 2021; Accepted 25 August 2021; Published 16 September 2021

Academic Editor: Ying-Ren Chien

Copyright (C) 2021 Jinzhong Song et al. This is an open access article distributed under the Creative Commons Attribution License, which permits unrestricted use, distribution, and reproduction in any medium, provided the original work is properly cited.

Based on one simulated skin-electrode electrochemical interface, some electrochemical characteristics based on skin-electrode contact pressure (SECP) for dry biomedical electrodes were analysed and applied in this research. First, 14 electrochemical characteristics including 2 static impedance (SI) characteristics, 11 alternating current impedance (ACI) characteristics and one polarization voltage $(\mathrm{PV})$, and $4 \mathrm{SECP}$ characteristics were extracted in one electrochemical evaluation platform, and their correlation trends were statistically analysed. Second, dry biomedical electrode samples developed by the company and the laboratory, including textile electrodes, Apple watch, AMAZFIT rice health bracelet 1S, and stainless steel electrodes, were placed horizontally and vertically on the "skin" surface of the electrochemical evaluation platform, whose polarization voltages were quantitatively analysed. Third, electrocardiogram (ECG) collection circuits based on an impedance transformation (IT) circuit for textile electrodes were designed, and a wearable ECG acquisition device was designed, which could obtain complete ECG signals. Experimental results showed SECP characteristics for dry electrodes had good correlations with static impedance and ACI characteristics and the better correlation values among $2-10 \mathrm{~Hz}$. In addition, polarization voltages in vertical state were smaller in horizontal state for dry biomedical electrodes, and polarization voltage of electrode pair (PVEP) values for Apple watch bottom was always smaller than ones for Apple watch crown and LMF-2 textile electrode. And the skin-electrode contact impedance of IT textile electrodes was less than the traditional textile electrodes.

\section{Introduction}

With the development of biomedical engineering technologies, the need for low-load, noninvasive medical supervision is becoming more and more prominent. People's health data can be obtained not only in hospitals but also at home by health monitoring equipment, whose own physical state can be known conveniently.
Biomedical signals (such as ECG, EMG, EEG, and EOG) were usually used to evaluate cardiovascular and other functions for human bodies widely, which was usually extracted by traditional wet paste electrodes $(\mathrm{Ag} / \mathrm{AgCl})$ nowadays. While it presents many problems $[1,2]$, for example, it needs to stick to the skin and easily causes allergic reaction. Recently, dry biomedical electrode, as one new biosensor, has attracted researchers' attention because they can obtain 
biomedical signals from human bodies with some advantages such as without glues, reused, convenient to find the location, and very useful for wearable health monitoring.

In recent years, many kinds of dry biomedical electrodes have been produced, such as stainless-steel dry electrode, noncontact electrodes, textile electrodes, microneedle electrodes, and smart bracelet electrode [2-4]. Stainless-steel dry electrode is mainly composed of a stainless-steel sheet plated with $\mathrm{Ag} / \mathrm{AgCl}$, which is used to extract ECG signals from human body surface according to touching the skin directly without conductive paste.

One kind of textile electrode was designed by Song et al. using Jacquard [3], and Ag was plated in the fiber in the weft direction; it was found that this method was more stable than the weaving structure and had a lower loosening effect. Pola and Vanhala used embroidery to make textile electrodes, which increased the skin-electrode contact area [5], and other kinds of textile electrodes were used to extract ECG signals $[6,7]$. Conductive fabrics were made of a variety of wire fibers, where the main metal elements used to transmit bioelectrical signals were $\mathrm{Ag}, \mathrm{AgCl}, \mathrm{AgNy}, \mathrm{AgCu}$, $\mathrm{Cu}, \mathrm{Ni}$, etc. Some textile electrodes were developed by Professor Tao Xiaoming's research team in Hong Kong Polytechnic University [8].

Microneedle-shaped dry electrodes had a variety of preparation methods: Silicon as an electrode material [9], multiwall carbon nanotubes (MWCNT) [10], and polymers for molds [11]. In order to ensure electrode conductivity and take into account biological compatibility, some metal materials were deposited on the electrode surface, which were mainly $\mathrm{Au}, \mathrm{Ag}$ and $\mathrm{Pt}$, and $\mathrm{IrO}$ [12].

However, dry biomedical electrodes are easy to form a big contact impedance and a large polarization voltage in skin-electrode interface when they were attached to the surface of human skins when collecting biomedical signals, and they often come across noise interference $[13,14]$. And a complete biomedical signal acquisition requires a good contact impedance (needing a good contact between electrode and skin), while it often causes people's discomfort $[14,15]$.

In recent years, some dry electrodes were used to collect ECG signals, and a number of products using dry electrode technologies had also been developed. Apple Watch Series 4 was on the market in 2018.09, whose important feature was the addition of ECG detection function. And ECG signals were detected from people's fingers and arms with two dry electrodes. AMAZFIT rice health bracelet $1 \mathrm{~S}$ was also on the market, with whom ECG signals could be can be recorded for 24 hours with dry electrodes.

Many electrochemical characteristics based on skinelectrode contact pressure (SECP) for dry biomedical electrodes were analysed and applied in this research. First, some electrochemical and skin-electrode contact pressure characteristics for dry electrodes were quantitatively extracted, and the correlation trends between electrochemical characteristics and SECP characteristics were statistically analysed. Second, dry biomedical electrode samples on the market developed by the company and the laboratory, including textile electrodes, apple watch, AMAZFIT rice health brace-

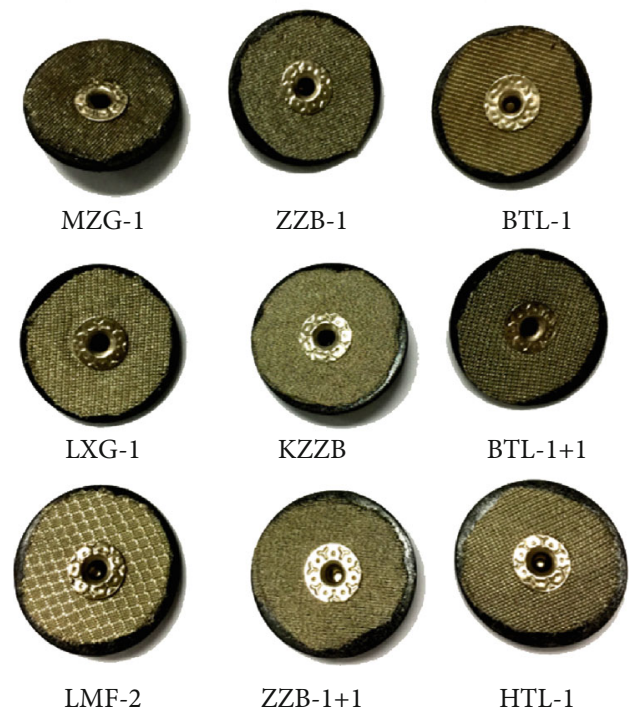

FIgURE 1: Textile electrode samples.
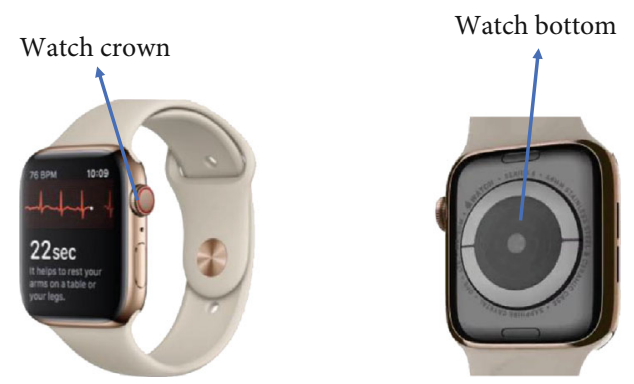

Figure 2: Watch bottom and watch crown for Apple Watch Series 4.

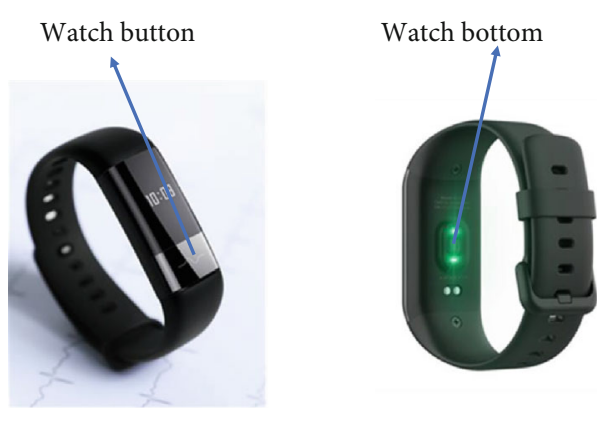

Figure 3: AMAZFIT rice health bracelet 1S.

let 1S, and stainless steel electrodes, were placed horizontally and vertically on the skin surface, whose polarization voltage was quantitatively analysed. Third, a wearable ECG acquisition device based on textile electrodes was developed: One kind of impedance transformation circuit for textile electrodes was designed, whose electrode-skin contact impedance was analysed compared with the traditional textile electrode, and a wearable collection device for ECG signals was designed, which could obtain the complete ECG signals. 


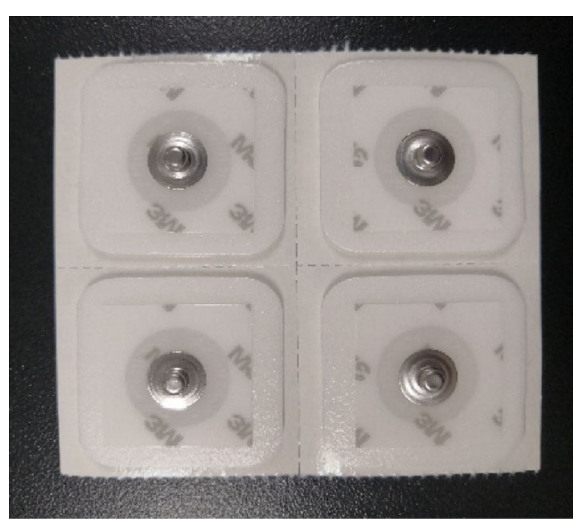

(a)

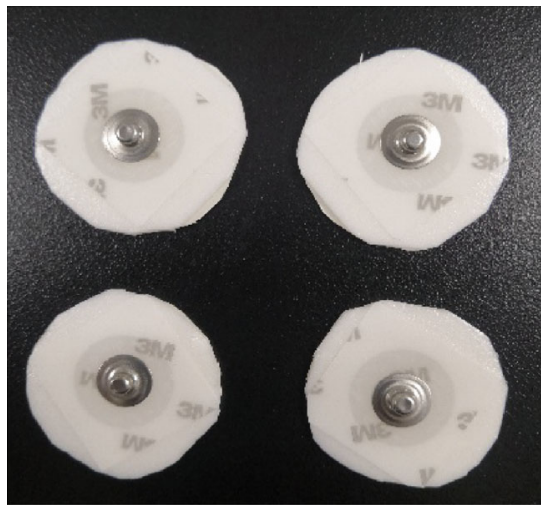

(b)

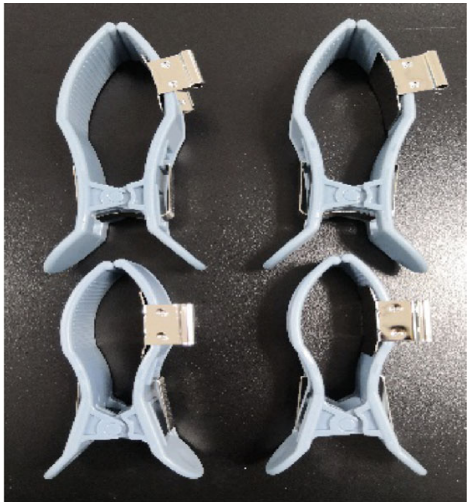

(c)

FIGURE 4: Traditional paste ECG electrodes in hospitals: (a) 3M ECG electrode; (b) 3M circle ECG electrode; (c) stainless-steel electrode.

\section{Materials and Methods}

2.1. Evaluation Platform. In this research, an electrochemical evaluation platform was set up to quantitatively evaluate dry biomedical electrodes [16], which could simulate the electrochemical interface between the dry biomedical electrodes and the skin surface.

2.2. Characteristic Settings for Dry Electrodes. Based on a passive electrochemical evaluation platform (PEEP) in the evaluation platform [16], the static impedance (SI) between electrode 1 and electrode 2 was measured by a CHI660 electrochemical workstation, and the SI values were measured by an impedance-time method. The parameters in this testing platform were set as follows: initial potential $0 \mathrm{~V}$, potential amplitude $0.01 \mathrm{~V}$, time $60 \mathrm{~s}$, and frequency $1000 \mathrm{~Hz}$. The dry electrodes in this research were intended for extraction of biomedical signals with amplitudes in the range of approximately $0 \sim 10 \mathrm{mV}$, so the potential amplitude was set to the $\mathrm{mV}$ level.

Alternating current impedance (ACI) values were measured by a CHI660 electrochemical workstation. The parameters in this testing platform were set as follows: initial potential $0.1 \mathrm{~V}$, high frequency $100 \mathrm{~Hz}$, low frequency $0.1 \mathrm{~Hz}$, and potential amplitude 0.1 .

Polarization voltage (PV) between electrode 1 and electrode 2 was measured by a digital mustimeter (Agilent Technologies U3402A).

Skin-electrode contact pressure (SECP) was measured by a pressure device, and the pressure range was set as $0 \sim 3 \mathrm{~N}$.

\subsection{Electrochemical Characteristics and Skin-Electrode Contact Pressure}

2.3.1. Electrochemical Characteristic Extraction. 14 electrochemical characteristics were extracted in the evaluation platform [16], which were 2 static impedance characteristics,

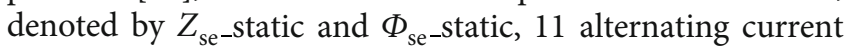
impedance characteristics, denoted by $Z_{\mathrm{ACI}-1} \sim Z_{\mathrm{ACI}-11}$, and one polarization voltage denoted by $U_{\mathrm{p}-\mathrm{se}}$.

\subsubsection{Skin-Electrode Contact Pressure (SECP) Characteristic} Extraction. For skin-electrode contact pressure characteris- tics, 4 characteristics were extracted [16], which including upper skin-electrode contact pressure (USECP, denoted by $F_{\text {USECP }}$ ), lower skin-electrode contact pressure (LSECP, denoted by $F_{\text {LSECP }}$ ), the difference between USECP and LSECP (SECP_D, denoted by $F_{\text {SECP_D }}$ ), and the sum of USECP and LSECP (SECP_S, denoted by $F_{\text {SECP_S }}$ ).

\section{Results and Discussion}

3.1. Dry Biomedical Electrode Samples. More and more wearable dry biomedical electrodes were used to obtain biomedical signals (such as ECG, EEG, and EOG) from the human body without skin pretreatment and conductive paste. Several types of dry biomedical electrodes had been produced, such as textile electrodes, stainless steel electrode, and smart bracelet electrode.

3.1.1. Textile Electrode Samples. Textile electrode as one type of dry biomedical electrodes was studied in this research, 9 kinds of conductive textile samples with different plating processes and different textile processes, whose definition was introduced in literature [16], were produced as shown in Figure 1.

\subsubsection{Dry Biomedical Electrode Samples in the Market. Apple} Watch Series 4 was on the market in 2018.09, whose important feature was the addition of ECG detection function. And ECG signals were detected from people's fingers and arms with two dry electrodes, which were watch bottom and watch crown for Apple Watch Series 4, as shown in Figure 2.

On the other hand, AMAZFIT rice health bracelet $1 \mathrm{~S}$ was on the market in 2018.09, with whom ECG signals could be recorded for 24 hours with dry electrodes. And their dry electrodes were watch button and watch bottom shown in Figure 3.

To evaluate different dry biomedical electrodes, some common ECG electrodes in the market were analysed, which included 3M ECG electrode, 3M circle ECG electrode with a diameter $2 \mathrm{~cm}$, and stainless-steel electrode as shown in Figure 4 . 


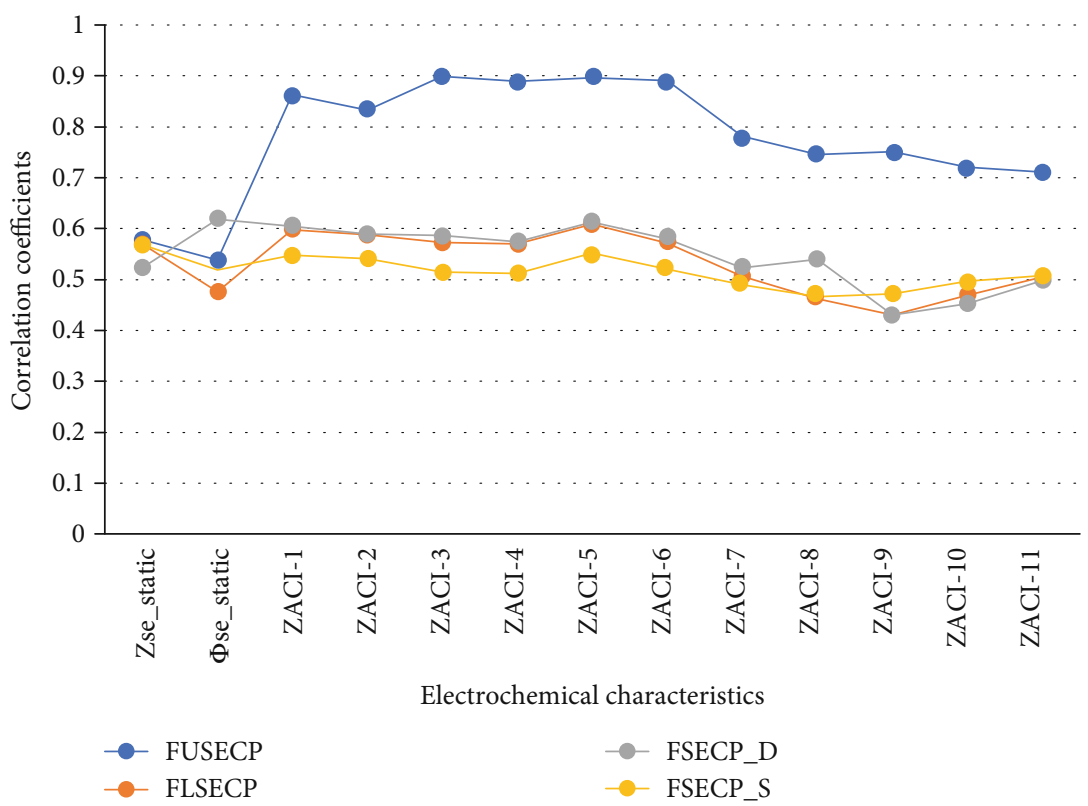

FIGURE 5: Trends of correlation coefficients between electrochemical and SECP characteristics.

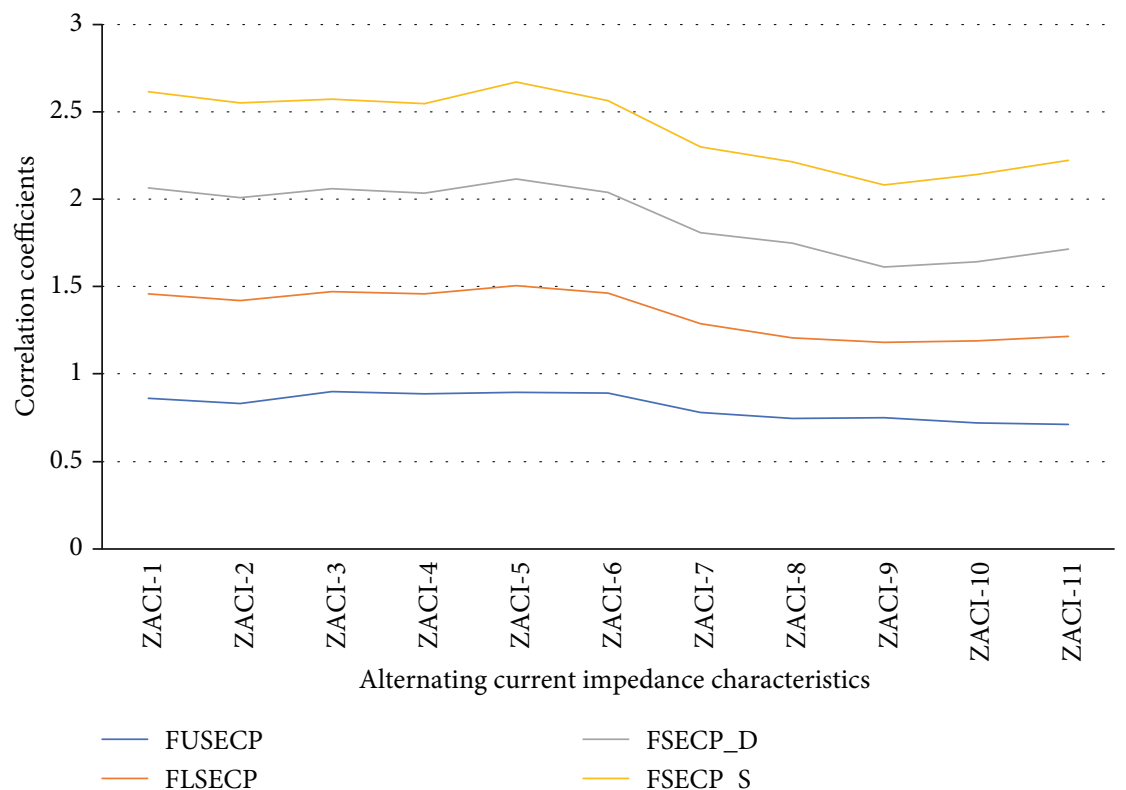

FIGURE 6: Four trends of correlation coefficients between alternating current impedance and SECP characteristics (note: $F_{\mathrm{USECP}}$ : +0 ; $\left.F_{\text {LSECP }}:+1 ; F_{\text {SECP_D }}:+2 ; F_{\text {SECP_S }}:+3\right)$.

3.2. Correlation Analysis. Based on 9 textile electrodes in Section 3.1.1, the trends of correlation coefficients between electrochemical characteristics and SECP characteristics versus for 4 skin-electrode contact pressure (SECP) characteristics are shown in Figure 5.

As could be seen from Figure 54 SECP characteristics (denoted by $F_{\text {USECP }}, F_{\text {LSECP }}, F_{\text {SECP_D }}$, and $F_{\text {SECP_S }}$ ), especially $F_{\text {USECP }}$, had good correlations with $Z_{\text {se }}$ static and alternating current impedance characteristics, and better correlation values occurred among $2-10 \mathrm{~Hz}\left(Z_{\mathrm{ACI}-3} \sim Z_{\mathrm{ACI}-6}\right)$; therefore, the influence of contact pressure on signal qualities should be considered when signals among $2-10 \mathrm{~Hz}$ were collected by textile electrodes.

As shown as Figure 6, each correlation coefficient curve for alternating current impedance characteristics in Figure 5 was shown separately. In order to describe the change trend of correlation coefficient better, the correlation coefficients of $F_{\text {USECP }}, F_{\text {LSECP }}, F_{\text {SECP_D }}$, and $F_{\text {SECP_S }}$ were plotted by adding $0,1,2$, and 3 , respectively, as shown in Figure 6.

With the increasing of frequency, the correlation coefficient between ACI and SECP characteristics decreased 


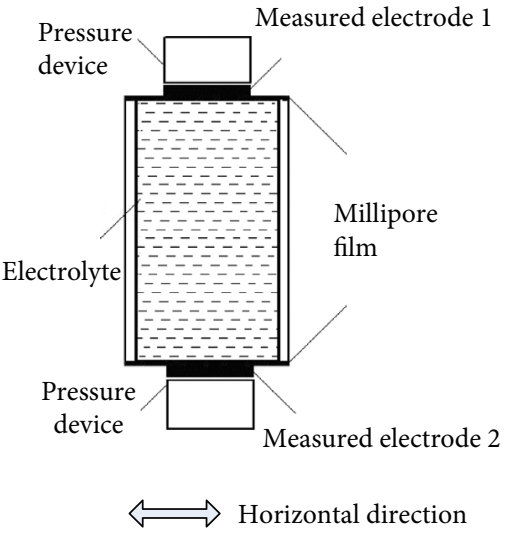

(a)

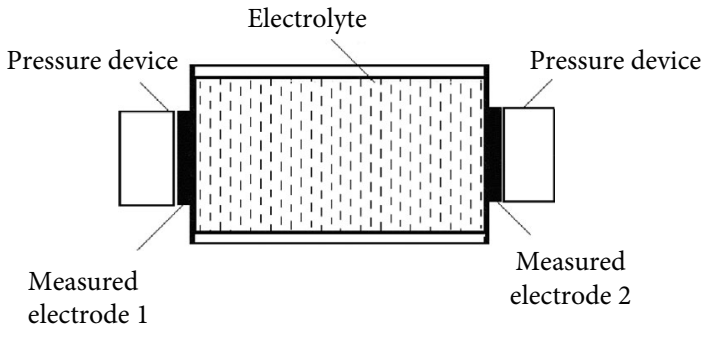

$\Uparrow$ Vertical direction

(b)

Figure 7: Horizontal and vertical model: (a) horizontal model; (2) vertical model.

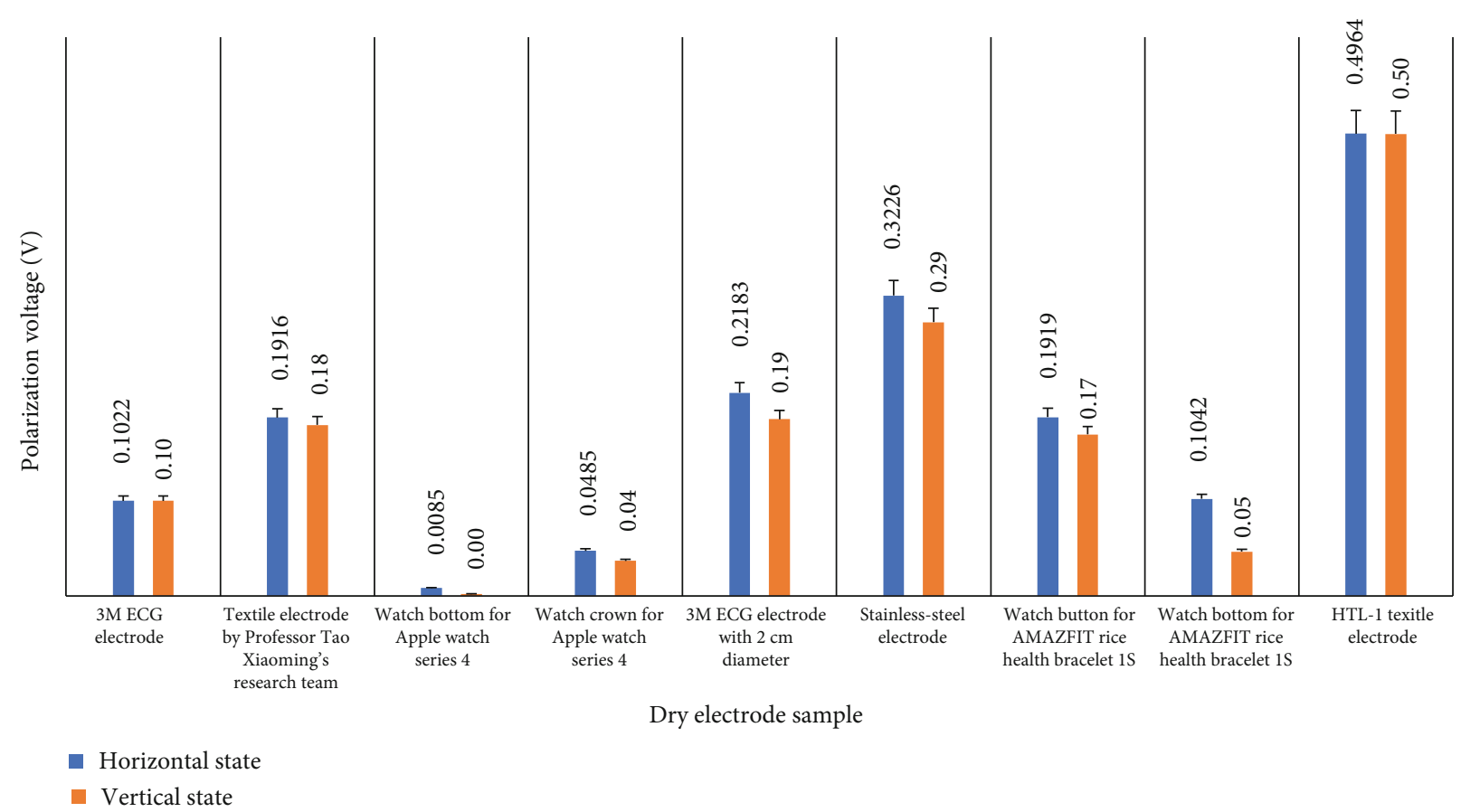

FIgURE 8: Polarization voltage in horizontal state and vertical state for different electrodes.

(especially above $10 \mathrm{~Hz}$ ), which showed that the quality of signals in low frequency could be improved by contact pressure.

Based on a variety of biomedical electrodes developed by the company and the laboratory, including 3M ECG electrode (shown in Figure 4), textile electrodes (shown in Figure 1), Apple watch (shown in Figure 2), AMAZFIT rice health bracelet $1 S$ (shown in Figure 3), and stainless steel electrodes (shown in Figure 4), these electrodes were placed horizontally and vertically on the skin surface, whose polarization voltages were quantitatively analysed as shown in Figure 7. The horizontal model is shown in Figure 7(a), and the electrode was placed horizontally on the skin surface, while the vertical model is shown in Figure $7(\mathrm{~b})$, and the electrode was placed vertically.
Based on above 9 kinds of biomedical electrodes, skinelectrode polarization voltages were measured in horizontal and vertical stations as shown in Figure 7, where the upper surface contact pressure in Figure 7 (a) and the left surface contact pressure in Figure $7(\mathrm{~b})$ were set as $40.12 \mathrm{cN}$, and the lower surface contact pressure in Figure 7 (a) and the right surface contact pressure in Figure $7(\mathrm{~b})$ were set as $5.11 \mathrm{cN}$.

Means and variances of polarization voltages in horizontal state and vertical state are shown in Figure 8.

As shown in Figure 8, polarization voltages in vertical state were smaller in horizontal state, which indicated that there was a significant difference for polarization voltages between the electrodes on the surface of the skin in the horizontal and vertical directions. 


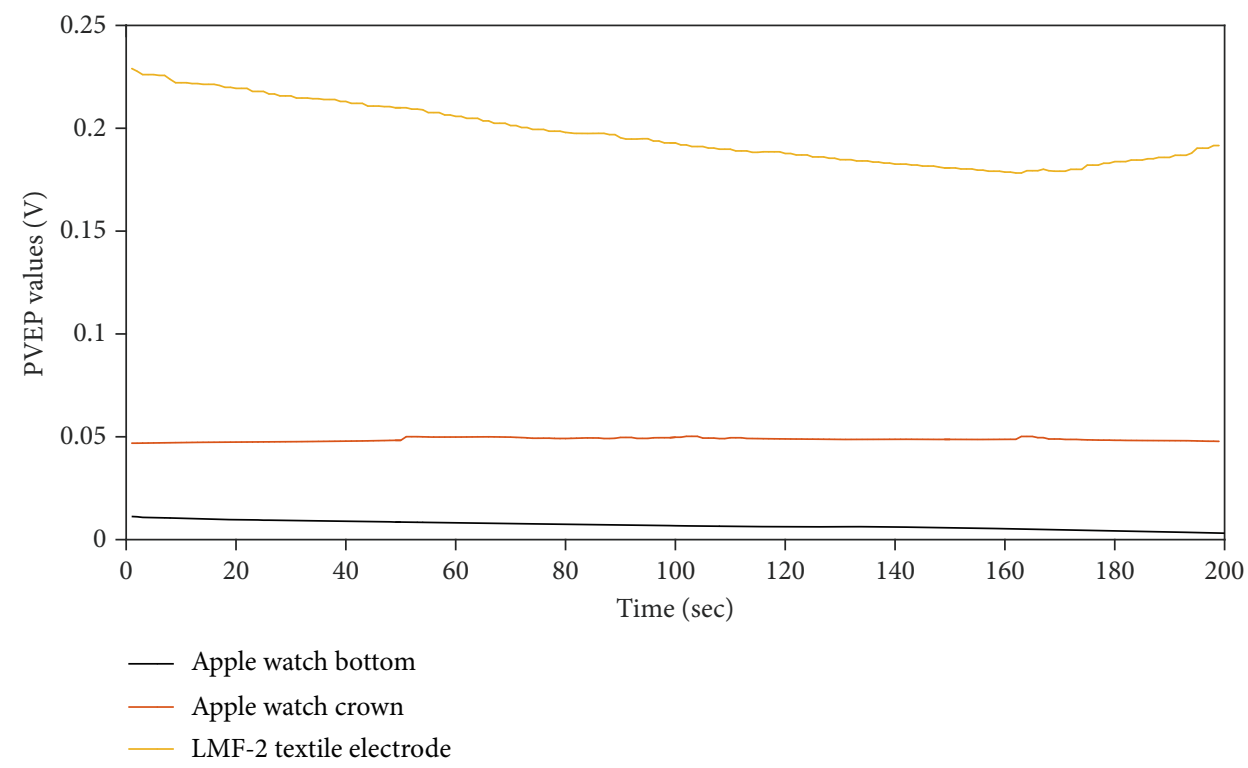

Figure 9: Polarization voltage for different dry electrodes.

The polarization voltage of electrode pairs (PVEP), defined as the sum of skin-electrode polarization voltage of electrode 1 and electrode 2 at the upper and lower Millipore film based on a passive electrochemical evaluation platform (PEEP) [16], was evaluated for the watch bottom and watch crown of Apple Watch Series 4 and one kind of conductive textile sample (LMF-2) from Qingdao Tianyin Textile Co., Ltd.

Based on PEEP [16], electrode 1 was the dry electrode sample, and electrode 2 was $3 \mathrm{M}$ electrode (model: 2560). $F_{\mathrm{USECP}}$ and $F_{\mathrm{LSECP}}$ were set as $40.12 \mathrm{cN}$ and $5.11 \mathrm{cN}$ separately. PVEP values for three kinds of dry electrodes (including Apple watch bottom, Apple watch crown, and LMF-2 textile electrode) are obtained as shown in Figure 9.

As shown in Figure 9, PVEP values showed a consistent change trend with time for three kinds of dry electrode samples, and PVEP values for Apple watch bottom were always smaller than ones for Apple watch crown and LMF-2 textile electrode.

\subsection{A Wearable Device for ECG Collecting}

3.3.1. Design of Impedance Transformation (IT) Textile Electrode. The textile electrode samples shown in Figure 1 were integrated into impedance transformation circuits to reduce the contact resistance between skin and electrodes. The circuit is shown in Figure 10. The voltage is changed from $200 \mathrm{k}$ to 100 , and the multiple is nearly 20,000 . As a result, an impedance transformation textile electrode (IT textile electrode) was produced (Figure 11).

In addition, the skin-electrode contact impedance of IT textile electrodes and traditional textile electrodes was quantitatively compared and analysed based on the electrochemical evaluation platform [16]. Experimental results showed that the electrode-skin contact impedance of IT textile electrodes was less than traditional textile electrodes, as shown in Figure 12.

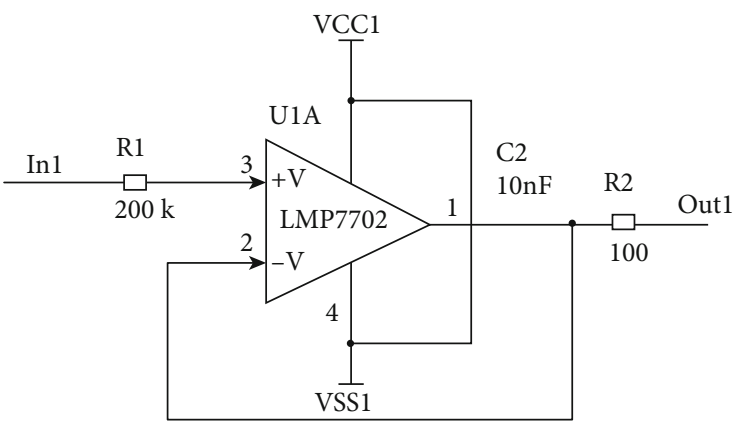

FIgURE 10: An impedance transformation circuit.

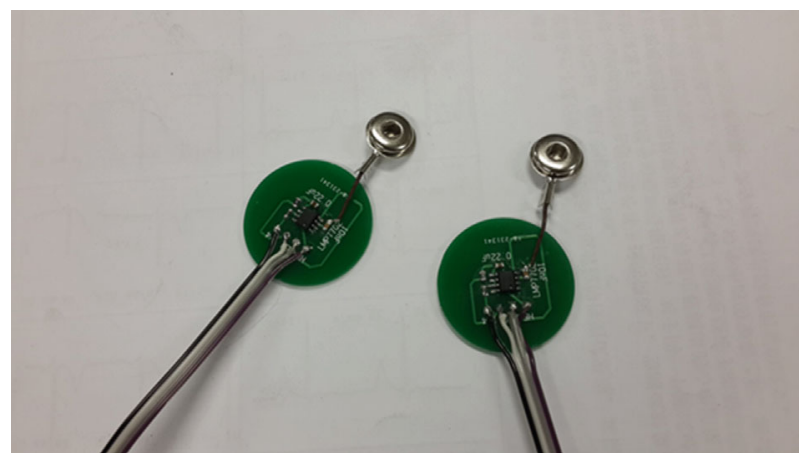

Figure 11: IT textile electrodes.

3.3.2. Chinese Aerospace ECG Lead System. Two-lead ECG signals were collected using Chinese aerospace ECG lead system including chest sword (CW) and chest axillary (CA), as shown in Figure 13.

3.3.3. ECG Conditioning Circuit. AD8221 was selected as an instrument amplifier, which had high common mode 


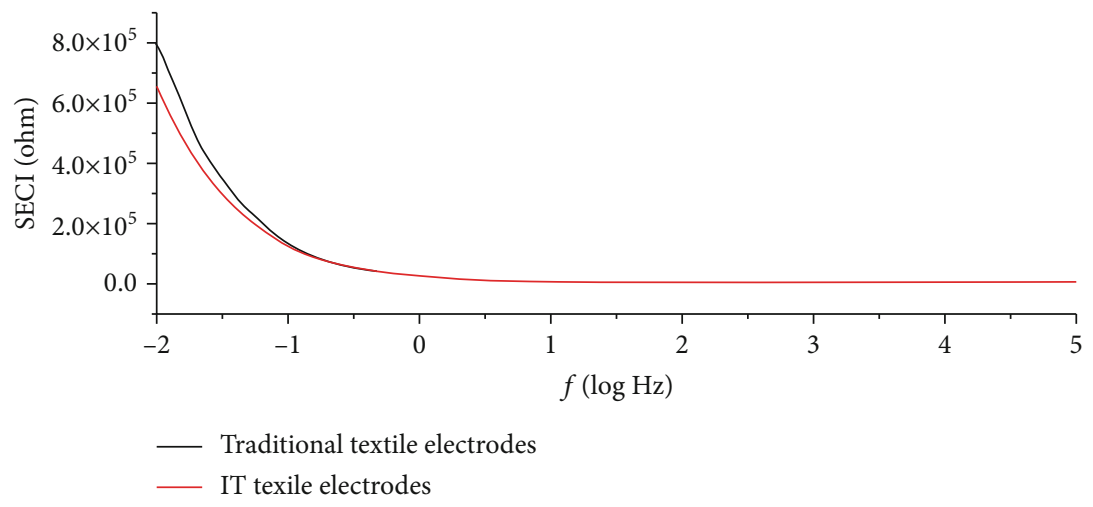

FIGURE 12: Contact impedance curve of IT and traditional textile electrode.

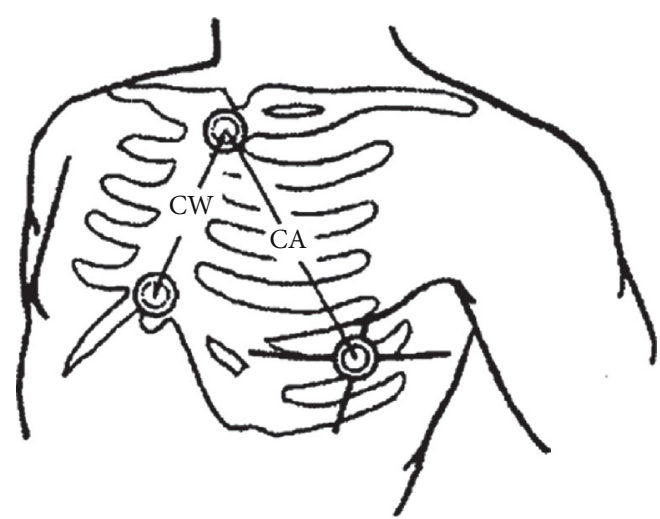

Figure 13: Chinese aerospace ECG lead system.

suppression ratio (CMRR). AD8221 gain (denoted by $G$ ) was programmed through the resistance $R_{G}$, which could be calculated by the following formula:

$$
R_{G}=\frac{49.4 k \Omega}{G-1}
$$

In this research, $G$ value (the gain of an instrument amplifier) was designed to be 200 when $R_{G}$ value was selected by $248 \Omega$.

3.3.4. Right Leg Drive Circuit. The right leg drive circuit was introduced to suppress the influence from common mode interference. A voltage follower was used to separate the drive circuit from the main circuit, and common signals were amplified 20 times and fed back to the human body.

3.3.5. Power Conversion Circuit. The ECG amplifier circuit was supplied by $\pm 5 \mathrm{~V}$, and a single power supply was used in this paper. $3.3 \mathrm{~V}$ reference level was provided for $\mathrm{AD}$ acquisition chips, which required high accuracy and less noise. A conversion circuit for $3.3 \mathrm{~V}$ reference level, $5 \mathrm{~V}$, and $-5 \mathrm{~V}$ are shown separately in Figures 14-16.

3.3.6. AD Acquisition and Wireless Communications. The ECG baseline level was not raised in the circuit design in this research for the miniaturization circuit design. Instead, AD7606 (positive and negative $\mathrm{AD}$ acquisition chips) was

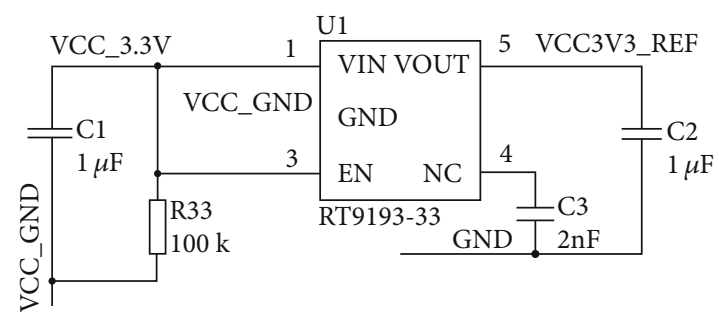

FIgURE 14: Conversion circuit for $3.3 \mathrm{~V}$ reference level.

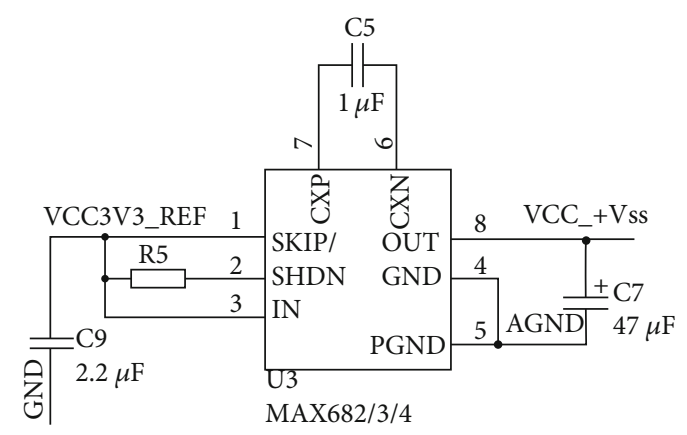

Figure 15: Conversion circuit (3.3 V to $5 \mathrm{~V})$.

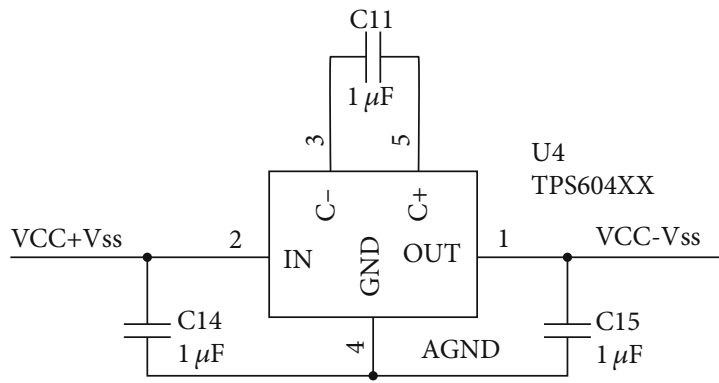

Figure 16: Conversion circuit ( $5 \mathrm{~V}$ to $-5 \mathrm{~V})$.

adopted, and Zigbee communication protocol was used for the wireless transmission.

3.3.7. Results for the Wearable ECG Acquisition Device. A wearable ECG acquisition device was developed based on a 


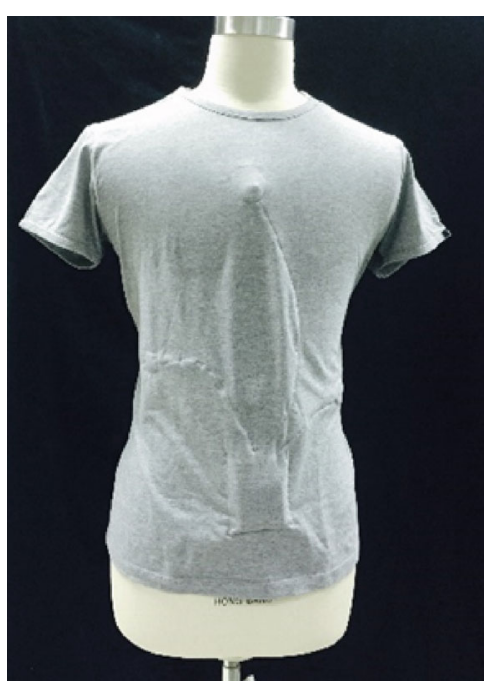

(a)

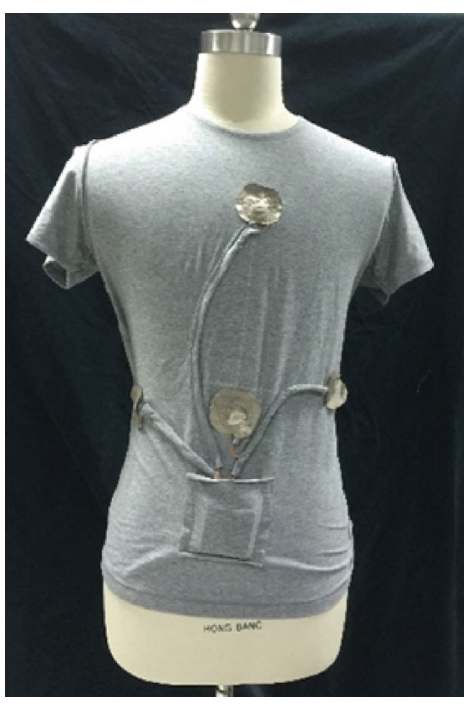

(b)

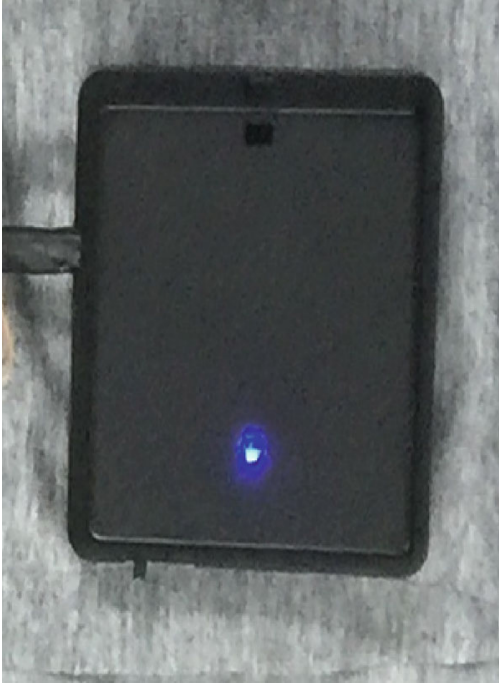

(c)

FIgURe 17: A wearable ECG collection device.

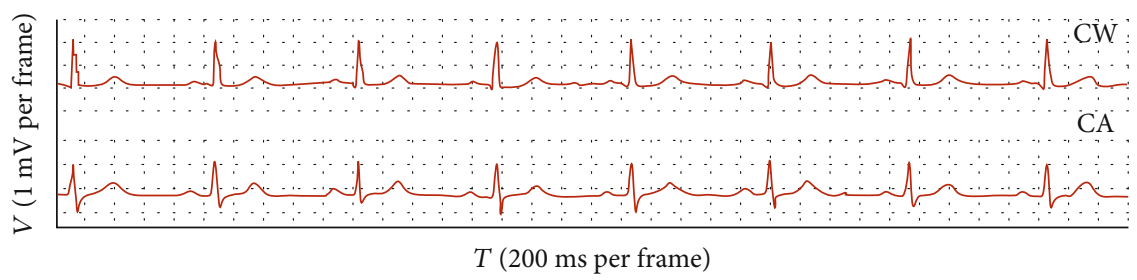

FIgURE 18: Two-lead ECG waveform.

garment integrating by IT textile electrodes, lead lines, and ECG collection circuit, which could extract two lead ECG signals in a low-load and unconstrained way.

The front of the garment for the wearable ECG signal acquisition is showed in Figure 17(a), and the reverse of the wearable ECG signal acquisition garment is showed in Figure 17(b), which combined IT electrodes, ECG lead lines, and ECG acquisition device. During the use for some subjects, if the garment is put on and the ECG acquisition device is turned on, collected ECG signals can be seen at the display terminal. The ECG acquisition device on the garment is showed in Figure 17(c).

Based on the above principle of ECG acquisition, it was proved by human experiments that the wearable ECG acquisition device developed in this paper could collect complete ECG waveform. The experimental results are shown in Figure 18.

\section{Conclusions}

Based on a variety of biomedical electrodes developed by the company and the laboratory, including 3M ECG electrode, textile electrodes, Apple watch, AMAZFIT rice health bracelet $1 \mathrm{~S}$, and stainless steel electrodes, correlations between electrochemical characteristics and skin-electrode contact pressure were analysed. Experimental results showed that
SECP characteristics for dry electrodes had good correlations with static impedance and ACI characteristics and the better correlation values among $2-10 \mathrm{~Hz}$. And polarization voltages in vertical state were smaller in horizontal state for dry biomedical electrodes.

In addition, one kind of textile electrode named by IT textile electrode was designed, whose electrode-skin contact impedance was analysed compared with traditional textile electrodes based on an electrochemical evaluation platform [16], and the electrode-skin contact impedance of IT textile electrodes was less than traditional textile electrodes. Then, a wearable device for ECG acquisition was designed, which could obtain the complete ECG waveform. Experimental results showed that the ECG signal acquisition based on IT textile electrode was feasible, which provided a basis for the production, evaluation, and application of dry biomedical electrodes. Issues such as cost, usability, and pandemic should be taken into account in the selection of devices and electrodes for actual health monitoring, experiments, sensing under daily activities, and health management.

\section{Data Availability}

The data that support the findings of this study are available from the corresponding author upon reasonable request. 


\section{Conflicts of Interest}

The authors declare that there are no conflicts of interest regarding the publication of this paper.

\section{Authors' Contributions}

Jinzhong Song and Tianshu Zhou contributed equally to this work.

\section{Acknowledgments}

This work was supported by the National Key Research and Development Program of China (No. 2018YFC0116901), the National Natural Science Foundation of China (No. 81771936, No. 81801796), the Fundamental Research Funds for the Central Universities (No. 2021FZZX00218), the Major Scientific Project of Zhejiang Lab (No. 2020ND8AD01), the Research Funds of China space medical engineering (2021SY54B0709, SMFA20C02), and the "Fei Tian" Foundation of Astronaut Center of China (2021SY54B0704).

\section{References}

[1] J. Song, H. Yan, Y. Li, and K. Mu, "Research on electrocardiogram baseline wandering correction based on wavelet transform, QRS barycenter fitting, and regional method," Australasian Physical \& Engineering Sciences in Medicine, vol. 33, no. 3, pp. 243-250, 2010.

[2] J. Song, H. Yan, G. Gong, Y. Zhang, Z. Cao, and L. Zhang, "Research progress of textile electrode technologies applied in electrocardiogram signal acquisition," Transducer and microsystem technologies, vol. 34, no. 10, pp. 4-7, 2015.

[3] H. Song, J. Lee, D. Kang et al., "Textile electrodes of jacquard woven fabrics for biosignal measurement," The Journal of the Textile Institute, vol. 101, pp. 768-770, 2010.

[4] J. Song, H. Yan, Z. Xu, X. Yu, and R. Zhu, "Myocardial ischemia analysis based on electrocardiogram QRS complex," Australasian Physical \& Engineering Sciences in Medicine, vol. 34, no. 4, pp. 515-521, 2011.

[5] T. Pola and J. Vanhala, "Textile electrode in ECG measurement," in Proceedings of 3rd international conference on intelligent sensors, pp. 635-639, Melbourne, 2007.

[6] J. Baek, J. An, J. Choi, K. S. Park, and S. H. Lee, "Flexible polymeric dry electrodes for the long-term monitoring of ECG," Sensors and Actuators A, vol. 143, pp. 423-429, 2008.

[7] J. Yoo, L. Yan, S. Lee, H. Kim, and H. J. Yoo, “A wearable ECG acquisition system with compact planar-fashionable circuit board-based shirt," IEEE Transactions on Information Technology in Biomedicine, vol. 13, no. 6, pp. 897-902, 2009.

[8] P. Xu, "Skin-electrode mechanical interaction and motion artifacts of textile electrodes for body surface ECG signal monitoring," in Textile material and textiles design, pp. 1-19, Donghua University, Shanghai, China, 2012.

[9] P. Griss, P. Enoksson, H. Tolvanen-Laakso, P. Merilainen, S. Ollmar, and G. Stemme, "Micromachined electrodes for biopotential measurements," Journal of microelectro-mechanical systems, vol. 10, no. 1, pp. 10-16, 2001.
[10] G. Ruffini, S. Dunne, L. Fuentemilla et al., "First human trials of a dry electro-physiology sensor using a carbon nanotube array interface," Sensors and Actuators A: Physical, vol. 144, pp. 275-279, 2008.

[11] W. Ng, H. Seet, K. Lee et al., "Micro-spike EEG electrode and the vacuum-casting technology for mass production," Journal of Material Processing Technology, vol. 209, no. 9, pp. 44344438, 2009.

[12] N. Dias, J. Carmo, A. da Silva, P. M. Mendes, and J. H. Correia, "New dry electrodes based on iridium oxide (IrO) for noninvasive biopotential recordings and stimulation," Sensors and Actuators A, vol. 164, pp. 28-34, 2010.

[13] Y. Chi, T. Jung, and G. Cauwenberghs, "Dry-contact and noncontact biopotential electrodes: methodological review," IEEE Reviews in Biomedical Engineering, vol. 3, pp. 106119, 2010.

[14] D. Duxi, S. Kim, N. Van Helleputte et al., "Correlation between electrode-tissue impedance and motion artifact in biopotential recordings," IEEE Sensors Journal, vol. 12, no. 12, pp. $3373-$ 3383, 2012.

[15] J. Song, H. Chen, H. Zhang, W. Chen, and X. Yu, "Detection methods for skin-electrode contact impedance of textile electrodes," Progress in modern biomedicine, vol. 15, no. 24, pp. 4777-4781, 2015.

[16] J. Song, Y. Zhang, Y. Yang et al., "Electrochemical modeling and evaluation for textile electrodes to skin," Biomedical Engineering Online, vol. 19, no. 1, pp. 1-27, 2020. 